\title{
Alterações metabólicas decorrentes do uso de contraceptivos hormonais: uma revisão integrativa de literatura
}

\author{
Metabolic alterations resulting from the use of hormonal contraceptives: an integrative literature \\ review
}

Alteraciones metabólicas derivadas del uso de anticonceptivos hormonales: revisión integradora de la literatura

\section{Resumo}

Introdução: o efeito adverso mais comum das pílulas anticoncepcionais orais combinadas é o sangramento. As mulheres também se queixam de náuseas, dores de cabeça, cólicas abdominais, sensibilidade mamária e aumento do corrimento vaginal ou diminuição da libido. No entanto, diferentes combinações de pílulas mostram diferentes tendências de obstrução da coagulação dos vasos (risco de trombose venosa), alterações nas vias metabólicas de lipídeos, na cascata de coagulação, na sensibilidade à insulina e nas propriedades vasoativas. Objetivo: explanar acerca das possíveis alterações metabólicas decorrentes do uso de contraceptivos hormonais. Metodologia: trata-se de uma pesquisa descritiva do tipo revisão integrativa da literatura, através do acesso online nas bases de dados PubMed, Scielo, CDSR, Google Scholar, BVS e EBSCO, no mês de outubro de 2021. Resultados e discussão: as usuárias de contraceptivos hormonais que contêm estrogênio estão sob maior risco de trombose venosa e tromboembolismo, haja vista que, em condições normais, o sistema da coagulação mantém em equilíbrio dinâmico os sistemas pró-coagulante e anticoagulante, e os estrogênios, principalmente o componente etinilestradiol, afetam esses dois sistemas de acordo com a dose. Além disso, as alterações na composição dos lipídeos plasmáticos produzidas pelos estrogênios caracterizam-se por um aumento das lipoproteínas de alta densidade (HDL), ligeira redução das lipoproteínas de baixa 
densidade (LDL) e diminuição dos níveis plasmáticos totais de colesterol. Ademais, os estrogênios aumentam a coagulabilidade do sangue. Conclusão: a maioria dos anticoncepcionais orais combinados apresentam um risco aumentado de trombose venosa e outras alterações metabólicas, sendo que o tamanho do efeito depende do progestágeno usado e da dose de etinilestradiol.

Palavras-chave: Anticoncepcionais; Estrogênio; Progesterona; Tromboembolismo; Alterações metabólicas.

\begin{abstract}
Introduction: The most common adverse effect of combined oral contraceptive pills is bleeding. Women also complain of nausea, headaches, abdominal cramps, breast tenderness, and increased vaginal discharge or decreased libido. However, different combinations of pills show different trends in obstruction of vessel coagulation (risk of venous thrombosis), changes in lipid metabolic pathways, coagulation cascade, insulin sensitivity and vasoactive properties. Objective: to explain about the possible metabolic changes resulting from the use of hormonal contraceptives. Methodology: this is a descriptive research of the integrative literature review type, through online access in the PubMed, Scielo, CDSR, Google Scholar, BVS and EBSCO databases, in October 2021. Results and discussion: the users of hormonal contraceptives that contain estrogen are at greater risk of venous thrombosis and thromboembolism, given that, under normal conditions, the coagulation system maintains the pro-coagulant and anticoagulant systems in dynamic balance, and estrogens, especially the ethinylestradiol component, affect these two systems according to dose. In addition, changes in the composition of plasma lipids produced by estrogens are characterized by an increase in high-density lipoproteins (HDL), a slight reduction in low-density lipoproteins (LDL) and a decrease in total plasma cholesterol levels. Furthermore, estrogens increase blood clotting. Conclusion: most combined oral contraceptives have an increased risk of venous thrombosis and other metabolic changes, and the size of the effect depends on the progestin used and the dose of ethinylestradiol.
\end{abstract}

Keywords: Contraceptives; Estrogen; Progesterone; Thromboembolism; Metabolic changes.

\begin{abstract}
Resumen
Introducción: El efecto adverso más común de las píldoras anticonceptivas orales combinadas es el sangrado. Las mujeres también se quejan de náuseas, dolores de cabeza, calambres abdominales, sensibilidad en los senos y aumento del flujo vaginal o disminución de la libido. Sin embargo, diferentes combinaciones de píldoras muestran diferentes tendencias en la obstrucción de la coagulación de los vasos (riesgo de trombosis venosa), cambios en las vías metabólicas de los lípidos, cascada de coagulación, sensibilidad a la insulina y propiedades vasoactivas. Objetivo: explicar los posibles cambios metabólicos derivados del uso de anticonceptivos hormonales. Metodología: se trata de una investigación descriptiva del tipo revisión integradora de la literatura, mediante acceso online en las bases de datos PubMed, Scielo, CDSR, Google Scholar, BVS y EBSCO, en octubre de 2021. Resultados y discusión: los usuarios de anticonceptivos hormonales que contienen estrógenos son con mayor riesgo de trombosis venosa y tromboembolismo, dado que, en condiciones normales, el sistema de coagulación mantiene en equilibrio dinámico los sistemas procoagulante y anticoagulante, y los estrógenos, especialmente el componente etinilestradiol, afectan estos dos sistemas según dosis. Además, los cambios en la composición de los lípidos plasmáticos producidos por los estrógenos se caracterizan por un aumento de las lipoproteínas de alta densidad (HDL), una ligera reducción de las lipoproteínas de baja densidad (LDL) y una disminución de los niveles de colesterol plasmático total. Además, los estrógenos aumentan la coagulación sanguínea. Conclusión: la mayoría de los anticonceptivos orales combinados tienen un mayor riesgo de trombosis venosa y otros cambios metabólicos, y la magnitud del efecto depende de la progestina utilizada y la dosis de etinilestradiol.
\end{abstract}

Palabras clave: Anticonceptivos; Estrógeno; Progesterona; Tromboembolismo; Cambios metabólicos.

\title{
1. Introdução
}

Atualmente, existem três tipos de pílulas anticoncepcionais orais (ACO): a combinação estrogênio-progesterona, apenas a progesterona e a pílula de uso contínuo ou prolongado. Aproximadamente $25 \%$ das mulheres de 15 a 44 anos que atualmente usam anticoncepcionais relataram usar a pílula como método de escolha. A pílula mais comumente prescrita é a pílula hormonal combinada com estrogênio e progesterona. A maioria das mulheres tomam ACO para prevenir a gravidez, mas $14 \%$ os usaram por razões não anticoncepcionais (Cooper et al., 2021).

A progesterona é a principal responsável pela prevenção da gravidez, sendo seu principal mecanismo de ação a prevenção da ovulação, ao inibir o desenvolvimento folicular e previnem a ovulação. O feedback negativo de progestagênio atua no hipotálamo para diminuir a frequência de pulso do hormônio liberador de gonadotrofina. Isso, por sua vez, diminui a secreção do hormônio folículo-estimulante (FSH) e diminuirá a secreção do hormônio luteinizante (LH). Se o folículo não estiver se desenvolvendo, não haverá aumento nos níveis de estradiol (o folículo produz o estradiol). O feedback negativo de 
progestágeno e a falta de feedback positivo de estrogênio na secreção de LH interrompem o pico de LH no meio do ciclo. Assim, sem o folículo desenvolvido e sem pico de LH para liberar o folículo, existe a prevenção da ovulação. O estrogênio tem algum efeito na inibição do desenvolvimento folicular por causa de seu feedback negativo na pituitária anterior, com diminuição da secreção de FSH; simplesmente não é tão proeminente quanto o efeito da progesterona. A atrofia endometrial induzida pela progesterona deve impedir a implantação, mas não há provas de que isso ocorra (Araújo et al., 2016).

A maioria dos efeitos colaterais dos ACOs são leves e desaparecem com o uso continuado ou com a mudança para outra formulação de pílula. $\mathrm{O}$ efeito adverso mais comum das pílulas anticoncepcionais orais combinadas é o sangramento. As mulheres também se queixam de náuseas, dores de cabeça, cólicas abdominais, sensibilidade mamária e aumento do corrimento vaginal ou diminuição da libido. No entanto, diferentes combinações de pílulas mostram diferentes tendências de obstrução da coagulação dos vasos (risco de trombose venosa), alterações nas vias metabólicas de lipídeos, na cascata de coagulação, na sensibilidade à insulina e nas propriedades vasoativas (Farias et al., 2016). Sob essa perspectiva, o objetivo deste estudo é explanar acerca das possíveis alterações metabólicas decorrentes do uso de contraceptivos hormonais.

\section{Metodologia}

Trata-se de uma pesquisa descritiva do tipo revisão integrativa da literatura, que buscou responder quais são as alterações metabólicas decorrentes do uso de anticonceptivos hormonais. A pesquisa foi realizada através do acesso online nas bases de dados National Library of Medicine (PubMed MEDLINE), Scientific Electronic Library Online (Scielo), Cochrane Database of Systematic Reviews (CDSR), Google Scholar, Biblioteca Virtual em Saúde (BVS) e EBSCO Information Services, no mês de outubro de 2021. Para a busca das obras foram utilizadas as palavras-chaves presentes nos descritores em Ciências da Saúde (DeCS): em inglês: "contraceptives", "progesterone", "estrogen", "thrombosis", "clotting", "metabolic alterations" e em português: "anticoncepcionais", "progesterona", "estrogênio", "trombose", "coagulação", "alterações metabólicas".

Como critérios de inclusão, foram considerados artigos originais, que abordassem o tema pesquisado e permitissem acesso integral ao conteúdo do estudo, publicados no período de 2011 a 2021, em inglês e português. O critério de exclusão foi imposto naqueles trabalhos que não estavam em inglês e espanhol, que não tinham passado por processo de Peer-View e que não abordassem as alterações metabólicas decorrentes do uso de anticoncepcionais hormonais. A estratégia de seleção dos artigos seguiu as seguintes etapas: busca nas bases de dados selecionadas; leitura dos títulos de todos os artigos encontrados e exclusão daqueles que não abordavam o assunto; leitura crítica dos resumos dos artigos e leitura na íntegra dos artigos selecionados nas etapas anteriores. Assim, totalizaram-se 20 artigos científicos para a revisão integrativa da literatura, com os descritores apresentados acima, dos últimos dez anos e em línguas portuguesa e inglesa.

\section{Resultados e Discussão}

Os estrogênios exercem vários efeitos metabólicos e cardiovasculares importantes e parecem ser responsáveis, em parte, pela manutenção da estrutura e função normais da pele e dos vasos sanguíneos nas mulheres (Batista et al., 2013). As alterações na composição dos lipídeos plasmáticos produzidas pelos estrogênios caracterizam-se por um aumento das lipoproteínas de alta densidade (HDL), ligeira redução das lipoproteínas de baixa densidade (LDL) e diminuição dos níveis plasmáticos totais de colesterol. Os níveis plasmáticos de triglicerídeos estão elevados. Além disso, os estrogênios aumentam a coagulabilidade do sangue. Foram relatadas inúmeras alterações nos fatores que influenciam a coagulação, inclusive aumento dos níveis circulantes dos fatores II, VII, IX e X, e diminuição da antitrombina III, devido, em parte, aos efeitos hepáticos já 
mencionados. Foram também observados aumentos nos níveis de plasminogênio e redução da adesão plaquetária (Almeida et al., 2017).

\section{- Trombose venosa}

As usuárias de contraceptivos hormonais que contêm estrogênio estão sob maior risco de trombose venosa e tromboembolismo, haja vista que, em condições normais, o sistema da coagulação mantém em equilíbrio dinâmico os sistemas pró-coagulante e anticoagulante, e os estrogênios, principalmente o componente etinilestradiol, afetam esses dois sistemas de acordo com a dose. Esses hormônios atuam diretamente na parede vascular, influenciando mudanças nos fatores que estimulam a disfunção endotelial. Essas transformações são favoráveis ao desenvolvimento de eventos tromboembólicos (Sousa et al., 2018).

Na maioria das mulheres, a fibrinólise (anticoagulação) é elevada tanto quanto a coagulação, mantendo o equilíbrio dinâmico com aumento dos níveis de produção e destruição do fibrinogênio (Bastos et al., 2014). Estudos antigos incluíam mulheres com distúrbios agora considerados contraindicações ao uso de contraceptivos hormonais com estrogênio: trombose prévia, doença vascular preexistente, doença coronariana, leucemia, câncer e traumatismo grave, e constataram que os contraceptivos orais (CO) de baixa dosagem atuais têm menor efeito mensurável sobre o sistema da coagulação, e os fatores fibrinolíticos aumentam na mesma proporção que os fatores pró-coagulantes. Ademais, os CO com menor dose de estrogênio (de 30 a $35 \mu \mathrm{g}$ de EE) reduzem o risco de tromboembolia quando comparados a CO de maior dose (50 $\mu \mathrm{g}$ de estrogênio) (Assis et al., 2017).

Um grande estudo dinamarquês mostrou, pela primeira vez, que os CO com $20 \mu$ de etinilestradiol estão associados a uma redução adicional de 18\% do risco de trombose em comparação com CO com 30 a $40 \mu \mathrm{g}$ após ajuste para duração do uso. No entanto, os CO que contêm apenas progesterona e o dispositivo intra-uterino (DIU) liberador de levonorgestrel não foram associados à trombose venosa (. Além disso, alguns estudos constataram aumento do risco de trombose venosa em usuárias de $\mathrm{CO}$ contendo os novos progestágenos desogestrel ou gestodeno associados a 20 a $30 \mu \mathrm{g}$ de etinilestradiol, em comparação a usuárias de levonorgestrel associado às mesmas doses de estrogênio, e o resultado foi controverso (Brandt et al., 2018). É provável que os vieses de "esgotamento de suscetíveis", a "seleção adversa" e o "viés de usuária saudável" expliquem o aumento aparente da trombose. A maioria dos casos de trombose venosa atribuíveis ao uso de CO ocorre durante os primeiros meses de uso (

A comparação de novas usuárias com mulheres que já tomam CO há algum tempo sem incidentes mostra o aumento aparente com o novo produto, que é artificial. Os médicos podem presumir que os novos fármacos são mais seguros e prescrevê-los de maneira seletiva para mulheres com fatores de risco. No entanto, a segurança dos anticoncepcionais hormonais combinados (CHCs) foi documentada por anos de acompanhamento, e a ocorrência de tromboembolismo venoso (TEV) possivelmente relacionado ao seu uso é rara na população jovem exposta a esses agentes. Porém. o equilíbrio entre os benefícios e riscos dos esteroides contraceptivos é geralmente positivo, em particular quando comparado com os riscos da gravidez (Guimarães, 2017).

\section{- Trombofilia}

Alterações no sistema da coagulação são detectáveis em todas as mulheres, inclusive naquelas que tomam CO de menor dose, bem como algumas delas têm predisposição genética à trombose quando estimuladas por gravidez ou administração de estrogênio exógeno, haja vista que, por exemplo, as mulheres com deficiência hereditária de antitrombina III, proteína $\mathrm{C}$ ou proteína $\mathrm{S}$ estão sob risco muito elevado de trombose durante a gravidez ou estrogenioterapia, mas representam uma proporção muito pequena de possíveis usuárias de CO (Morais et al., 2019). Uma variação bem mais comum, a do fator V 
de Leiden, existe em uma taxa de 3 a 5\% da população branca, que determina a mutação de um aminoácido na proteína do fator $\mathrm{V}$, inibindo a clivagem da proteína pela proteína $\mathrm{C}$ ativada (etapa essencial na manutenção do equilíbrio entre a coagulação e a fibrinólise) (Farias et al., 2016).

Uma mutação semelhante é encontrada na posição 20210 no gene da protrombina e é descrita como protrombina G20210A. Essa mutação ocorre em 3\% da população europeia e também está fortemente associada à trombose venosa em usuárias de CO. Há muitas outras condições genéticas que predispõem à trombose, e a gravidez é um desafio ainda maior para mulheres com defeitos hereditários da anticoagulação (Cooper et al., 2021). Sob essa perspectiva, uma mulher com problema venoso durante o uso de CO deve ser submetida a avaliação completa, que deve incluir, no mínimo, a pesquisa dos níveis de antitrombina III, proteína $\mathrm{C}$ e proteína $\mathrm{S}$, resistência à proteína $\mathrm{C}$ ativada, homocisteína sérica, mutação do fator $\mathrm{V}$ de Leiden, mutação da protrombina G20210A e teste para anticorpo antifosfolipídio, Ademais, recomenda-se com veemência o rastreamento de mulheres com história pessoal ou familiar de trombose venosa profunda antes do início da contracepção hormonal com estrogênio ou durante a gravidez. Não devem ser administrados contraceptivos com estrogênio - pílula, adesivo ou anel - às mulheres com diagnóstico de fator $\mathrm{V}$ de Leiden. Além disso, não se deve supor que a contracepção hormonal seja a única razão do episódio de trombose (Brito et al., 2011).

\section{- Cardiopatia isquêmica}

A cardiopatia isquêmica e o acidente vascular encefálico (AVE) foram as principais causas de morte atribuídas ao uso de CO no passado. Sabe-se que os principais determinantes de risco são a idade avançada e o tabagismo, haja vista que as mulheres que fumavam 25 cigarros ou mais por dia apresentavam o aumento de 30 vezes do risco de infarto agudo do miocárdio (IAM) se usassem CO quando comparadas a mulheres não tabagistas e que não usavam CO. Por isso, o uso de CO tornou-se mais seguro em razão de a maioria das mulheres usar pílulas de baixa dosagem e os médicos fazerem uma prescrição seletiva, excluindo mulheres com importantes fatores de risco cardiovasculares (Ferreira et al., 2019).

Acredita-se que a associação ao infarto do miocárdio envolve uma aceleração da aterogênese, devido à redução da tolerância à glicose, aos níveis diminuídos de HDL, aos níveis elevados de LDL e a um aumento da agregação plaquetária. Além disso, a facilitação do espasmo arterial coronariano pode desempenhar uma função em algumas dessas pacientes. Haja vista que o componente pró-gestacional dos contraceptivos orais diminui os níveis de HDL em proporção à atividade androgênica da progestina, o efeito final dependerá da composição específica da pílula utilizada e da suscetibilidade da paciente aos efeitos específicos. Além disso, o risco de infarto não está aumentado em usuárias que abandonaram o uso de contraceptivos orais (Ribeiro et al., 2018).

\section{- Acidente Vascular Encefálico (AVE)}

Na década de 1970, o uso de CO parecia estar relacionado com o risco de AVE hemorrágico e trombótico. No entanto, esses estudos não levaram em conta os fatores de risco preexistentes, haja vista que as evidências atuais não apontam risco de AVE em mulheres saudáveis e usuárias de pílulas de baixa dosagem (Ministério da Saúde, 2017). Além disso, a doença de Moyamoya, um tipo raro de insuficiência vascular cerebral, está relacionada ao uso de CO, sobretudo em tabagistas. Ademais, as habitantes de países em desenvolvimento tiveram um pequeno aumento aparente do risco; no entanto, esse achado foi atribuído a fatores de risco existentes não detectados (Lima et al., 2017).

Outro estudo realizado na Europa, além de constatar menor risco de AVE com pílulas de baixa dosagem do que com as antigas pílulas de maior dosagem, concluiu que o risco era menor se a pressão arterial da paciente fosse verificada antes do início do uso de CO. Outrossim, as mulheres tabagistas, hipertensas e diabéticas correm maior risco de doença cardiovascular, sendo usuárias ou não de $\mathrm{CO}$, sendo o questionamento importante se o uso de $\mathrm{CO}$ de baixa dosagem eleva o risco e, nesse 
caso, em que grau. O estudo da OMS descrito anteriormente oferece algumas informações importantes: tabagistas em uso de $\mathrm{CO}$ apresentaram risco de AVE isquêmico (trombótico) aumentado em sete vezes, em comparação a tabagistas que não usavam CO; além disso, mulheres hipertensas tiveram aumentado em 10 vezes o risco com uso de CO, mas um aumento de cinco vezes sem uso de CO (Oliveira et al., 2021).

Da mesma maneira, um estudo na Dinamarca constatou que o risco de AVE em mulheres diabéticas era cinco vezes maior, mas se tornava 10 vezes maior se usassem $\mathrm{CO}$, no entanto, esses dados não foram limitados a CO com baixa dosagem de estrogênio. Os dados sugerem que, embora o risco seja determinado principalmente pelo distúrbio predisponente (hipertensão, diabetes ou tabagismo), pode ser elevado pelo uso de $\mathrm{CO}$, mesmo de baixa dosagens. O risco de AVE isquêmico e de infarto agudo do miocárdio foi maior em hipertensas usuárias de $\mathrm{CO}$ de estrogênio-progesterona que em mulheres não usuárias de CO. Por isso, é prudente a conduta atual na América do Norte de limitar o uso de contraceptivos hormonais com estrogênio por mulheres com mais de 35 anos de idade às não tabagistas sem outros fatores de risco para doença vascular (Olsen et al., 2018).

\section{- Hipertensão arterial}

Acredita-se que o estrogênio exógeno contribui na ativação do sistema reninaangiotensina-aldosterona, causando efeitos hipertensores mesmo em pequenas dosagens; e que o uso combinado com a drospirenona reduz esses efeitos. Vias de administração sem passagem pelo fígado e uso do progestágeno isolado mostraram resultados promissores na redução dos efeitos sobre a pressão arterial. Com as antigas pílulas de alta dosagem, esperava-se que até 5\% das pacientes tivessem pressão arterial superior a 140/90 mmHg. Acredita-se que o mecanismo seja uma elevação do substrato renina, induzido pelo estrogênio, em mulheres suscetíveis. Já as pílulas de baixa dosagem atuais causam efeitos mínimos sobre a pressão arterial, mas é aconselhável a vigilância contínua da pressão arterial para se detectar a resposta idiossincrásica ocasional (Souza et al., 2016).

Ademais, os estrogênios também alteram a estrutura e a função das glândulas suprarrenais. Quando administrados por via oral ou em altas doses, os estrogênios aumentam a concentração plasmática da $\alpha 2$ globulina que se liga ao cortisol (globulina de ligação dos corticosteroides). As concentrações plasmáticas podem ser mais de duas vezes os níveis observados em indivíduos não tratados, e a excreção urinária de cortisol livre apresenta-se elevada (Ferreira et al., 2019).

\section{- Metabolismo da glicose}

O estrogênio oral isolado não tem efeito adverso sobre o metabolismo da glicose, mas os progestágenos são antagonistas da insulina. As formulações antigas de $\mathrm{CO}$ com doses maiores de progestágenos produziam anormalidades nos testes de tolerância à glicose com níveis de insulina elevados na paciente média. $\mathrm{O}$ efeito sobre o metabolismo da glicose, semelhante àquele sobre os lipídios, está relacionado à potência androgênica dos progestágenos e à dose (Oliveira et al., 2021).

\section{- Metabolismo dos lipídios}

Sabe-se que androgênios e estrogênios têm efeitos antagônicos sobre a lipase hepática, uma enzima hepática essencial para o metabolismo lipídico. Os estrogênios reduzem as lipoproteínas de baixa densidade (LDL) e elevam as lipoproteínas de alta densidade (HDL), alterações que podem reduzir o risco de aterosclerose. Assim, os androgênios e os progestágenos com forte ação androgênica podem opor-se a essas modificações benéficas, reduzindo os níveis de HDL e elevando os níveis de LDL, e os estrogênios elevam os níveis de triglicerídios (Trindade et al., 2019).

As formulações de baixa dosagem têm efeito adverso mínimo sobre os lipídios, e as novas formulações (com desogestrel e norgestimato como progestágeno) produzem mudanças que podem ser benéficas pela elevação da HDL e 
diminuição da LDL. Embora os valores médios de um grande grupo mostrem apenas pequenas alterações lipídicas com os CO atuais, às vezes os efeitos podem ser exagerados em determinada paciente. As anormalidades durante o tratamento são mais prováveis nas mulheres cujos valores lipídicos estejam acima da média antes do tratamento (Cooper et al., 2021).

\section{- Outros efeitos metabólicos}

Os COs podem causar alterações em uma grande variedade de proteínas sintetizadas pelo fígado. O estrogênio contido nos CO eleva a globulina de ligação do hormônio tireoidiano circulante, o que afeta as provas de função tireoidiana baseadas nessa ligação, aumenta os níveis de tiroxina total (T4) e diminui a captação de tri-iodotironina (T3) (resin uptake). Os resultados das provas de função tireoidiana de rotina, como a dosagem de T4 livre e os exames com iodo radioativo, são normais (Oliveira et al., 2021).

\section{Conclusão}

Portanto, nota-se que a maioria dos anticoncepcionais orais combinados apresentam um risco aumentado de trombose venosa e outras alterações metabólicas, sendo que o tamanho do efeito depende do progestágeno usado e da dose de etinilestradiol. Por isso, deve ser prescrito o anticoncepcional oral combinado com a menor dose possível de etinilestradiol e boa adesão, ou seja, $30 \mu \mathrm{g}$ de etinilestradiol com levonorgestrel. A avaliação dessas diferentes tendências pode desempenhar um papel importante na escolha da pílula mais segura ao iniciar o uso da pílula. Além disso, notou-se que anticoncepcionais orais contendo doses mais altas de estrogênio ( $>30 \mu \mathrm{g}$ ) com levonorgestrel (um progestágeno) ou contendo acetato de ciproterona ou drospirenona como progestágeno estão associados a maior risco de tromboembolismo venoso e demais alterações metabólicas do que a pílula anticoncepcional oral com $30 \mu \mathrm{g}$ de estrogênio e utilização de levonorgestrel como progestágeno.

\section{Referências}

Almeida, A. P., et al. (2017). Efeitos Colaterais E Alterações Fisiológicas Relacionadas Ao Uso Contínuo De Anticoncepcionais Hormonais Orais. Rev. Eletrôn. Atualiza Saúde, 5 (5), 85-93.

Araújo, A. B. R., et al. (2016). Anticoncepcionais hormonais contendo apenas progestágenos e seus principais efeitos. Brasilian Jourmal of Surgery and Clinical Research, 15 (1), 75-81.

Assis, R. S. G. F. (2017). Contraceptivos Orais e o risco Trombótico. Trabalho de conclusão de curso: UNIC, Primavera do Leste- MT.

Bastos, M., et al. (2014). Contraceptivos orais combinados: trombose venosa. Cochrane Database of Systematic Reviews, 3 (108), 1-54.

Batista, M. N., et al. (2013). Planejamento Familiar: Métodos e Contraceptivos. Revista Científica Multidisciplinar Núcleo do Conhecimento, 1 (7), 29-41.

Brandt, G. P., et al. (2018). Anticoncepcionais hormonais na atualidade: um novo paradigma para o planejamento familiar. Revista Gestão \& Saúde, 18 (1), 54-62.

Brito, M. B., et al. (2011). Contracepção hormonal e sistema cardiovascular. Atualização Clínica, 96 (4), 1-11.

Cooper, D. B., et al. (2021). Pílulas anticoncepcionais orais. StatPearls, 5 (8), 1-10.

Farias, M. R., et al. (2016). Utilização e acesso a contraceptivos orais e injetáveis no Brasil. Revista de Saúde Pública, 3 (8), 1-10.

Ferreira, L. F., et al. (2019). O uso da pílula anticoncepcional e as alterações das principais vias metabólicas. Feminas, 47 (7), $426-32$.

Guimarães, M. A. (2017). Trombose associada ao uso de contraceptivos hormonal oral: revisão da literatura. Trabalho de conclusão de curso de Biomedicina- Centro Universitário de Brasília: UNICEUB, Brasília.

Lima, A. C., et al. (2017). Influência de anticoncepcionais hormonais e ocorrência de acidente vascular cerebral: revisão integrativa. Revista Brasileira de Enfermagem, 70 (3), 647-655.

Ministério da Saúde. (2017). Anticoncepção hormonal oral. Disponível em: https://bvsms.saude.gov.br/bvs/publicacoes/0102assistencia2.pdf. Acessado em: 10 de outubro de 2021 .

Morais, L. X., et al. (2019). Tromboembolismo venoso relacionado ao uso frequente de anticoncepcionais orais combinados. FASEM Rechst, 8 (1), 91-125. 
Research, Society and Development, v. 10, n. 13, e481101321628, 2021

(CC BY 4.0) | ISSN 2525-3409 | DOI: http://dx.doi.org/10.33448/rsd-v10i13.21628

Oliveira, R. P. C., et al. (2021). O anticoncepcional hormonal via oral e seus efeitos colaterais para as mulheres. Revista Artigos., 28 (3), 12-21.

Olsen, J. M., et al. (2018). Práticas contraceptivas de mulheres jovens: inquérito domiciliar no Município de São Paulo, Brasil. Cadernos de Saúde Pública, 34 (12), 43-59.

Ribeiro, M. C. C., et al. (2018). Efeitos dos diferentes anticoncepcionais hormonais nos valores de pressão arterial da mulher. Revista Brasileira de Enfermagem, 71 (8), 1-11.

Sousa, I. C., et al. (2018). A trombose venosa profunda como reação adversa do uso contínuo de anticoncepcionais orais. Revista de Divulgação Científica Sena Aires, 7 (1), 54-65.

Souza, G. G., et al. (2016). Conhecimento e uso de anticoncepcionais hormonais: o que é certo ou errado? Temas em Saúde, 16 (4), 1-12.

Trindade, R. E., et al. (2019). Uso de contracepção e desigualdades do planejamento reprodutivo das mulheres Brasileiras. Ciência e Saúde Coletiva, 3 (7), 114. 